\title{
Face of the Myocardial Infarction and Diabetes: Epidemiological and Clinical Aspects in the Cardiology Department of the University Hospital Ignace Deen about 46 Cases
}

\author{
Samoura Aly ${ }^{*}{ }^{\infty}$, Samoura Sana1, Camara Abdoulaye1, Barry Ibrahima Sory ${ }^{1}$, \\ Soumaoro Morlaye ${ }^{1}$, Koivogui Diarra1, Doumbouya Mohamed', Toure Demba ${ }^{2}$, \\ Balde Elhadj Yaya1, Beavogui Mariama1, Balde Mamadou Dadhi', Conde Mamady \\ ${ }^{1}$ Cardiac Service, Ignace Deen CHU, Conakry, Guinea \\ ${ }^{2}$ Pneumology Service, Ignace Deen CHU, Conakry, Guinea \\ Email: *samouraaly01@gmail.com
}

How to cite this paper: Aly, S., Sana, S., Abdoulaye, C., Sory, B.I., Morlaye, S., Diarra, K., Mohamed, D., Demba, T., Yaya, B.E., Mariama, B., Dadhi, B.M. and Mamady, C. (2019) Face of the Myocardial Infarction and Diabetes: Epidemiological and Clinical Aspects in the Cardiology Department of the University Hospital Ignace Deen about 46 Cases. World Journal of Cardiovascular Diseases, 9, 692-697. https://doi.org/10.4236/wjcd.2019.99062

Received: August 9, 2019

Accepted: September 21, 2019

Published: September 24, 2019

Copyright () 2019 by author(s) and Scientific Research Publishing Inc. This work is licensed under the Creative Commons Attribution International License (CC BY 4.0).

http://creativecommons.org/licenses/by/4.0/

(c) (7) Open Access

\begin{abstract}
The purpose of this study was to describe myocardial infarction in diabetics at the Cardiology Department Ignace Deen. Introduction: New international recommendations defined myocardial infarction, any coronary syndrome accompanied by a modification of cardiac troponin even minimal Tc or Ic [1]. Then for diabetes is defined by the World Health Organization (WHO) a blood glucose greater than $1.10 \mathrm{~g} / \mathrm{l}$ and/or capillary blood glucose greater than $2.26 \mathrm{~g} / \mathrm{l}$ after eight hours of fasting [2]. Patients and Methods: Were eligible for both male and female patients at any age admitted to cardiology for myocardial infarction and receiving an ECG echocardiogram; A biological evaluation including blood glucose and troponin Tc or Ic. Discussion: The risk of myocardial infarction is clearly increased in diabetics and is a determinant of coronary heart disease severity and a potential test for other cardiovascular risk factors at the Ignatius Deen Cardiac Department with a frequency of $16.08 \%$. Our result is lower than that of DIOUMM et al. in the Fanh cardiology department of Dakar, Senegal [6], which should reduce the frequency of $22.3 \%$. The high frequency of this pathology in the study DIOUMM is related to the size of their sample on the one hand and secondly the duration of the study, the service capacity on the other hand that could be explained. Conclusion: Diabetes mellitus is a scary medium and long-term disease that causes complications. In addition to infectious and metabolic complications, heart and coronary heart attacks are becoming more common. The tare is dominated by atherosclerosis, which must be prevented through the analysis of diabetic responsibility, with cardiovascular control of risk factors and
\end{abstract}


monitoring of myocardial ischemia, and good cooperation between diabetes specialists, cardiologists and patients.

\section{Keywords}

Diabetic, Myocardial Infarction, University Hospital, Ignace Deen

\section{Introduction}

The new international guidelines define myocardial infarction in the presence of acute coronary syndrome, accompanied by an elevation (even minimal) of cardiac troponin Tc or Ic [1].

And as for diabetes, according to the World Health Organization (WHO), a state of chronic capillary hyperglycemia is greater than $1.26 \mathrm{~g} / \mathrm{l}$ and/or vein 1,10 $\mathrm{g} / \mathrm{l}$ after the eighth of fasting [2].

Today, diabetes mellitus is a truly global epidemic. The number of diabetic patients is estimated at 382 million worldwide, of which more than $95 \%$ are type 2. The lengthening of life expectancy, sedentary life and regime changes explain the increase in the number of type 2 diabetes. The WHO predicts 592 million cases of diabetes by 2035 , that is $10.1 \%$ of the world's population [3].

Cardiovascular and especially coronary heart diseases represent the main cause of morbidity and mortality among diabetics (75\% of mortality). Indeed, diabetes doubles the overall risk of coronary heart disease, cardiovascular death and nonfatal MI [4].

Coronary involvement is more frequent, earlier and more serious in the diabetic than in the general population. Epidemiological data (DCCT and UKPDS) clearly show that diabetes increases the risk of coronary heart disease by 2 to 3 in men and 3 to 5 in women. The Framinghan survey found that diabetes was a risk factor.

Coronary artery is dependent on major factors such as hypertension, hyperlipidemia and smoking. In the multicenter CORONAFRIC study in 13 African countries, coronary arteries caused the onset of 50 cardiovascular diseases. However, its severity is due to the severity and multiplicity of atherosclerosis, but especially to the association with other lesions. Coronary artery disease is the leading cause of death in type 2 diabetics and does not spare diabetics Type 1 patients over 30 years of age (50\% of deaths from cardiac complications) [5]. In Guinea, no study has yet been carried out on the association between myocardial infarction and diabetes, hence the interest of this study, which aimed to study the epidemiological and paraclinical aspects of myocardial infarction and diabetes in order to determine their frequency, and to identify factors related to the occurrence of a myocardial infarction in diabetics of the department of cardiology, Ignace Deen.

\section{Patients and Method}

The study took place in the cardiology department of Ignace Deen University 
Hospital. The cardiology department is located to the right of the CHU and goes to the Conakry Nation Palace. It comprises 33 hospitals divided into two hospitalization zones and a cardio-intensive care room. It is composed of six cardiologists, three assistants and an assistant (DES), as well as doctors (DES) in specialization training. Patients are admitted either by outpatient consultation or by reference to other health structures in the country. This was a prospective descriptive study of the cross-sectional type (i.e., a scientific research method that involves observing the clinical characteristics of a population as a whole or a representative sample at a given time) given from January 1, 2018 to October 31, 2018. The sample was comprehensive and consisted of 46 . We selected 46 patients meeting the inclusion criteria out of 286 .

\subsection{Inclusion Criteria}

All patients of both sexes and at any age admitted to the cardiology department for myocardial infarction and diabetes are associated or associated with another cardiovascular pathology and benefiting from ECG echocardiography were eligible for the protocol. of sub-monthly heart disease, a biological assessment including fasting blood glucose at eight hours, troponin Tc or Ic, serum creatinine who died in search of morbidity.

\subsection{Criteria for Non-Inclusion}

Included in this study are all patients of both sexes and all patients admitted to the cardiology department for myocardial infarction and diabetes isolated or associated with other cardiovascular conditions and not benefiting from echocardiogram ECG, seeking underlying heart disease, an assessment that includes eight-hour fasting glucose, troponin Tc, or Ic.

\subsection{Limits and Constraints of the Study}

- The absence of certain means of paraclinical exploration (coronarography, arteriography, ECG exercise) and the non-completion of certain examinations in our context did not allow us to certify certain etiological diagnoses.

- The impossibility of performing a coronarography, a stressed arteriography, the ECG in our context is a factor of underestimation of the cardiac and macro-vascular disorders of our study.

\section{Results}

We included 46 patients. The average age was 62.8 , with a female prevalence of 1.19. During the study period, 286 patients consulted in the department, including 46 cases of myocardial infarction and diabetes, had a frequency of $16.08 \%$. Diabetes risk factors were dominated by $80 \%$ hypertension followed by $65 \%$ smoking. More than half of the patients were poly factorial. The symptomatology was dominated by atypical pain in $63 \%$ and asymptomatic patients by $36.95 \%$. All of our patients had a history of cardiovascular disease. No; of our patients 
had not had thrombolysis.

Biologically; fasting blood glucose was reached in all our patients and all patients had taken troponin. A quarter of our patients had a helpless function. The resting electrocardiogram revealed anomalies of ventricular polarization of the ST segment and the $\mathrm{T}$ wave in $3 / 4$ of the patents. We also found the necrotic wave $\mathrm{Q}$.

Cardiac Focal Doppler revealed a left ventricular ejection fraction of $69.56 \%$; kinetic disorders accounted for $86.56 \%$ of cases; reported hypokinesia 8, 69\% and dyskinesia $86 ; 95 \%$. None of our patients had coronary angiography. Regarding the management, among our patients, no one benefits from angioplasty or coronary surgery. Medical treatment was retained in all our patients. The most used molecules were BASIC in all patients, ADO (oral antidiabetic agent) $73.9 \%$, insulin $26 \%$ and diuretics (cycle and thiazide diuretics) at $34.78 \%$.

The most common complications were $34.79 \%$ heart failure with killip 4 stages, $17.39 \%$ and $17.39 \%$ cardiogenic shock and a large intermittent monomorphic extrasystole with a frequency of $15.21 \%$. The evolution was favorable in $60.86 \%$ of the cases but mortality was observed in $17.39 \%$ of the cases.

\section{Discussion}

The risk of myocardial infarction is significantly increased in diabetics and is a determining factor in the severity of coronary heart disease and potentiates other cardiovascular risk factors in cardiac patients of Ignatius Deen with a frequency of $16.08 \%$. Our result is lower than that of DIOUM M et al., At Fann cardiology department in Dakar, Senegal [6], which reported a frequency of $22.3 \%$. The high frequency of this pathology in the study of DIOUM M et al compared to our study could be explained by the duration of the study on the one hand and on the other hand by the capacity to host the service. In our series, we noted a female predominance of 25 cases or $54.30 \%$ with a sex ratio of 0.84 . Our data contrast with those of DIOUM $\mathrm{M}$ et al., the high frequency of female sex could be explained by the increase in smoking and alcoholism among unoccupied women, against the majority of the Guinean population is female. The mean age of our patients was \pm 62.8 years with extremes of 34 to over 81 years and the age group presented was the oldest between 51 and 65 years old with a frequency of $54.34 \%$. The symptomatology was dominated by atypical chest pain at $63 \%$ and asymptomatic patients at $36.95 \%$. Our result is similar to that of DIOUM M et al. The management was medical in all our patients because of the lack of equipment of the technical platform and was wired to $17.39 \%$ at stage 4 killip. This can be explained by several mechanisms: an alteration of the nerve fibers of oneself for the heart and the vessels, this alteration resulting in abnormalities of the cardiac rhythm of control or the vascular function responsible for ischemia responsible for a delay of diagnosis and management [7]. Diabetic cardiomyopathy is primarily a metabolic cardiomyopathy with abnormal use of substrates. Fatty acids are physiologically the main substrates used by the heart, providing $60 \%-70 \%$ of ATP requirements. However, diabetes is also accompanied by 
hyperglycemia, an increase in circulating fatty acids, a sharp increase in their use and cardiac oxidation. These, accumulated in cardiomyocytes, may be responsible for true lipotoxicity. We also note abnormalities in calcium homeostasis that can cause impaired excitation-contraction coupling [8]. Our results are similar to those of MERZOUK F, KHATOURI A study on acute coronary syndrome and type 2 diabetes involving 244 cases at the Avicenne Military Clinic The risk of myocardial infarction is considerably increased in diabetics and is a determining factor in the severity of coronary heart disease and potentiates other cardiovascular risk factors in cardiac patients of Ignace Deen with a frequency of $16.08 \%$. Our result is lower than that of DIOUM M et al., A Fann cardiac department in Dakar, Senegal [6], which reported a frequency of $22.3 \%$. The high frequency of this pathology in the study of DIOUM $\mathrm{M}$ et al compared to our study could be explained by the duration of the study on the one hand and on the other hand by the capacity to host the service. In our series, we noted a female predominance of 25 cases or $54.30 \%$ with a sex ratio of 0.84 . Our data contrast with those of DIOUM M et al, the high frequency of female sex could be explained by the increase in smoking and alcoholism among unoccupied women, against the majority of the Guinean population is female. The mean age of our patients was \pm 62.8 years with extremes of 34 to over 81 years and the age group presented was the oldest between 51 and 65 years old with a frequency of $54.34 \%$. The symptomatology was dominated by atypical chest pain at $63 \%$ and asymptomatic patients at $36.95 \%$. Our result is similar to that of DIOUM M et al. The management was medical in all our patients because of the lack of equipment of the technical platform and was wired to $17.39 \%$ at stage 4 killip. This can be explained by several mechanisms: an alteration of the nerve fibers of oneself for the heart and the vessels, this alteration resulting in abnormalities of the cardiac rhythm of control or the vascular function responsible for ischemia responsible for a delay of diagnosis and management. One by one factors of 2 mufflers) [7]. Diabetic cardiomyopathy is primarily a metabolic cardiomyopathy with abnormal use of substrates. Fatty acids are physiologically the main substrates used by the heart, providing $60 \%-70 \%$ of ATP requirements. However, diabetes is also accompanied by hyperglycemia, an increase in circulating fatty acids, a sharp increase in their use and cardiac oxidation. These, accumulated in cardiomyocytes, may be responsible for true lipotoxicity. We also note abnormalities in calcium homeostasis that can cause impaired excitation-contraction coupling [8]. Our results are similar to those of MERZOUK F, KHATOURI A study on acute coronary syndrome and type 2 diabetes involving 244 cases at the military clinic Avicenne Hospital Cardiology Service in Marrakech in 2012 [9]. Who should report in their series $27.97 \%$ of diabetic patients are admitted to stages 3 and 4 of Killip. With regard to evolution, it was favorable in $60.86 \%$ of cases, but mortality was observed with a frequency of $17.39 \%$. This mortality is certainly important but corresponds to the results of the works already published in Africa which confirm the severe prognosis. Myocardial infarction and diabetes are reported generally in the literature and conferring on them the cha- 
racter of a clear public health problem. The large mortality observed in our series could be explained by: the delays of consultation of the patients, the care limited by the insufficiency of the technical plateau and especially by the low socio-economic level of the population.

\section{Conclusion}

Diabetes mellitus is a formidable disease in the medium and long term because of these complications. In addition to infectious and metabolic complications, heart attacks and Coronaries are becoming more common. They are dominated by atherosclerosis and their consequences lead to excess mortality that should be avoided through effective management of diabetes, control of cardiovascular risk factors and screening for silent myocardial ischemia. It is a close collaboration between diabetologists, cardiologists and educated patients, aware of the risks involved, diligent consultation and effective support.

\section{Conflicts of Interest}

The authors declare no conflicts of interest regarding the publication of this paper.

\section{References}

[1] European Cardiovascular Society ESC (2012) The Vascular Cardiology Reference Edition 2016 KB. 120.

[2] World Health Organization (WMO). http://www.who.int

[3] Diabetes. IDF: IDF Diabetes Atlas I. 6th Edition. http://www.santediabete.org/fr/le-diabete-en-chiffres

[4] Emerging Risk Factors Collaboration, et al. (2010) Diabetes Mellitus, Fasting Blood Glucose Concentration, and Risk of Vascular Disease: A Collaborative $\mathrm{Me}$ ta-Analysis of 102 Prospective Studies. The Lancet, 375, 2215-2222. https://doi.org/10.1016/S0140-6736(10)60484-9

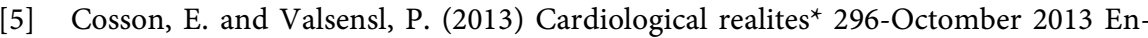
docrinology-Diabetology, service CRNH-dF.

[6] Dioum, M., et al. (2016) Coronary Disease in the Diabetic Patient Clinical, Paraclinical and Therapeutic Difficulties in the Cardiology Department of Fann in Dakar, Senegal.

[7] Ziegler, D., Zentai, C., Perz, S., et al. (2006) Selective Contribution of Diabetes and Other Cardiovascular Risk Factors to Cardiac Autonomic Dysfunction in the General Population. Experimental and Clinical Endocrinology \& Diabetes, 114, 153-159.

[8] Strom, J. (2014) Diabetic Heart Disease: Insights from Cardiac Mechanics. Journal of the American Society of Echocardiography, 27, 489-492. https://doi.org/10.1016/j.echo.2014.03.008

[9] Merzouk, F. and Khatouri, A. (2014) Acute Coronary Syndrome and Diabetes Type 2 Study of 244 Cases. Cardiology Service. Military Hospital Avicenna of Marrakech. 